\title{
MOLECULAR DATA CONFIRM THE SPECIES STATUS OF NEOECHINORHYNCHUS PERSONATUS AND N. YAMAGUTII (ACANTHOCEPHALA, NEOECHINORHYNCHIDAE) FROM THE ATLANTIC AND PACIFIC GREY MULLETS (TELEOSTEI, MUGILIDAE)
}

\author{
V. Sarabeev ${ }^{1 \star}$, Ie. Tkach ${ }^{1}$, R. A. Sueiro ${ }^{2}$, J. Leiro ${ }^{2}$ \\ ${ }^{1}$ Department of Biology, Zaporizhzhia National University, Zhukovskogo, 66, Zhaporizhzhia, 69063 Ukraine \\ ${ }^{2}$ Departamento de Microbiología y Parasitología, Instituto de Investigación y Análisis Alimentarios, \\ Universidad de Santiago de Compostela, Santiago de Compostela, 15782 Spain \\ ${ }^{*}$ Corresponding author \\ E-mail addres:vosa@ext.uv.es,volodimir.sarabeev@gmail.com
}

Molecular Data Confirm the Species Status of Neoechinorhynchus personatus and N. yamagutii (Acanthocephala, Neoechinorhynchidae) from the Atlantic and Pacific Grey Mullets (Teleostei, Mugilidae). Sarabeev, V., Tkach, Ie., Sueiro, R. A., Leiro, J. - Neoechinorhynchus is known to be the most diverse acanthocephalan taxon with worldwide distribution; its species are characterized by uniformity of anatomical organization. The taxonomic status of Neoechinorhynchus agilis s. l. from grey mullets was recently reviewed that resulted in description of two new species, Neoechinorhynchus (Hebesoma) personatus and Neoechinorhynchus (Hebesoma) yamagutii. In the current study,18S rRNA partial gene sequences were obtained to molecularly characterize $N$. (N.) agilis, $N$. (H.) personatus and N. (H.) yamagutii from grey mullets, Chelon labrosus and Mugil cephalus, in the North-East Atlantic, the Japan, Azov and Mediterranean Seas. The universal (F-566 and R-1200) and specific eukaryotic set primers were used to amplify specimens from each species of Neoechinorhynchus. It has been found that three species can be clearly recognized using universal primers, which allow to obtain sequences of 590 to $664 \mathrm{bp}$ in length. The genetic divergences estimated between three species sequenced here were relatively high, at ranged between 2.08 and $6.57 \%$. Phylogenetic analysis demonstrates that the studied species of Neoechinorhynchus from grey mullet fish share common ancestor, despite their different geographic location, and are closely related. The terminal position of $N$. (N.) agilis and $N$. (H.) personatus on the evolution tree and the low genetic divergence found between them suggests the recent emergence of this group and that the colonization of the North-East Atlantic and the Mediterranean regions could represent a single event. Our phylogenetic analysis, which included several species of the subgenera Neoechinorhynchus and Hebesoma, showed that the latter is a polyphyletic taxon.

Key words: 18S rRNA partial gene sequences, Chelon labrosus, Mugil cephalus, Neoechinorhynchus agilis, Neoechinorhynchus personatus, Neoechinorhynchus yamagutii. 


\section{Introduction}

The taxonomic status of Neoechinorhynchus agilis s. 1. (Rudolphi, 1819) from grey mullets (Mugilidae) across localities in the North-East Atlantic and the North-West Pacific areas was recently reviewed by Tkach et al. (2014). This taxonomic review performed on morphological features showed that there were three different species of Neoechinorhynchus occurring in mullet hosts, two of those were recorded in the Atlantic and one in the Pacific waters (Neoechinorhynchus (Neoechinorhynchus) agilis (Rudolphi, 1819) and Neoechinorhynchus (Hebesoma) personatus Tkach, Sarabeev et Shvetsova, 2014, Neoechinorhynchus (Hebesoma) yamagutii Tkach, Sarabeev et Shvetsova, 2014, respectively). Morphological identification of Neoechinorhynchus spp. from grey mullets is based on the combination of a set of specific characters describing the number of hypodermal nuclei, features of the hooks, lemnisci and genital organs. While the conventional microscopy applied to identification of Neoechinorhynchus is complicated because of high species diversity and specific morphology of the group, which commonly requires experienced researcher, accurate taxonomic identification is essential for biological and ecological studies. DNA barcoding techniques are the most technological and precise methods for the identification of a specimens bulk to the genus and species level (Morand, 2018). Biodiversity assessment method of metabarcoding uses a short section of DNA from a standardized region of the genome (Hebert et al., 2003). In the present study, we test the pair of the universal primers, F-566 and R-1200 to amplify variable regions of $18 \mathrm{~S}$ rRNA gene (Hadziavdic et al., 2014) of three species of Neoechinorhynchus from grey mullets. We also designed a set of specific primers that were used to obtain longer sequences of the studied species and to perform comparative phylogenetic analysis. Some regions of the $18 \mathrm{~S}$ rRNA gene are highly conserved, others are variable or even highly variable (Hadziavdic et al., 2014); thus this gene can provide a basis for resolving relationships at the genus and species levels of studied here acanthocephalan parasites.

Although Neoechinorhynchus is the most diverse acanthocephalan group with 121 known species, a few taxa of the genus were analysed using molecular data (Amin, 2013; Smales, 2013; Pinacho-Pinacho et al., 2014; Tkach et al., 2014; Melo et al., 2015; Gautam et al., 2018). There is much fewer data available on 18S rRNA sequences for Neoechinorhynchus spp. Malyarchuk et al. (2014) investigated molecular phylogenetic relationships of six Neoechinorhynchus species from fishes collected in freshwater localities of North-East Asia using DNA sequences of two genes, cytochrome CO1 of the mitochondrial DNA and 18S rRNA. Two additional almost complete $18 \mathrm{~S}$ rRNA sequences were obtained by Near et al. (1998) and García-Varela \& Nadler (2005) for Neoechinorhynchus (Neoechinorhynchus) crassus Van Cleave, 1919 and Neoechinorhynchus saginatus Van Cleave et Bangham, 1949, respectively, in their studies of acanthocephalan phylogeny. Finally, three sequences of the target regions of $18 \mathrm{~S}$ rRNA for Neoechinorhynchus (Neoechinorhynchus) pseudemydis Cable et Hopp, 1954, N. (N.) cylindratus (Van Cleave, 1914) and N. (N.) dimorphospinus Amin et Sey, 1996 were found as deposited in GenBank (table 1).

Here, 18S rRNA partial gene sequences of $N$. (N.) agilis, $N$. (H.) personatus and $N$. (H.) yamagutii were obtained from grey mullet hosts in localities across the North-East Atlantic and North-West Pacific regions, allowing us to molecularly characterize them, reveal interspecific relationships and test the systematic position within the genus.

\section{Materials and methods}

Material collection

Grey mullets were collected from five localities in the North-East Atlantic and the Japan, Azov and Mediterranean Seas (table 1). In the current study, acanthocephalan parasites were sampled from two fish species, Chelon labrosus (Risso) and Mugil cephalus L. Fish intestine and pyloric caeca were examined for parasites within the day of capture, or refrigerated, and surveyed for infections with acanthocephalans under a stereomicroscope. The worms were isolated from the intestine, washed in $0.8 \%$ saline water and after eversion of the proboscis fixed and stored in $70 \%$ or absolute ethanol prior to examination. The specimens of Neoechinorhynchus collected in this study were identified as $N$. (N.) agilis, N. (H.) personatus and N. (H.) yamagutii based on morphological features and host-geographic information (Tkach et al., 2014).

DNA extraction, amplification, sequencing and analysis

Specimens of acanthocephalans were placed individually in tubes and sonicated in TE buffer for several seconds. Then sodium dodecylsulfate and proteinase $\mathrm{K}$ were added to the mixture and digested 2 hours at $37^{\circ} \mathrm{C}$ with subsequent phenol-chloroform deproteinization, isopropanol precipitation, and ethanol washing. DNA was analysed to estimate its purity and concentration with the NanoDrop ND-1000 Spectrophotometer (NanoDrop Technologies, USA). PCR amplification was performed as previously described by Leiro et al. (2000) and De Felipe et al. (2017). The universal eukaryotic primers (F-566:5'-CAG CAG CCGCGGTAATTC C-3' and R-1200:5'-CCC GTGTTGAGTCAA ATT AAG C-3') proposed by Hadziavdic et al. (2014) were used to amplify V4 and V5 variable region of $18 \mathrm{~S}$ rRNA gene as regions with high taxonomic information. Based on available sequences of $18 \mathrm{~S}$ rRNA gene in GenBank (www.ncbi.nlm.nih.gov/genbank/) and new ones obtained in the current study for specimens of Neoechinorhynchus, the specific primer set (FNeo1086:5'-ATA GCCATG CAT GTG CAG TT-3' and RNeo1086:5-GCC TTGCGACCATACTCC C-3') was designed and optimized by 


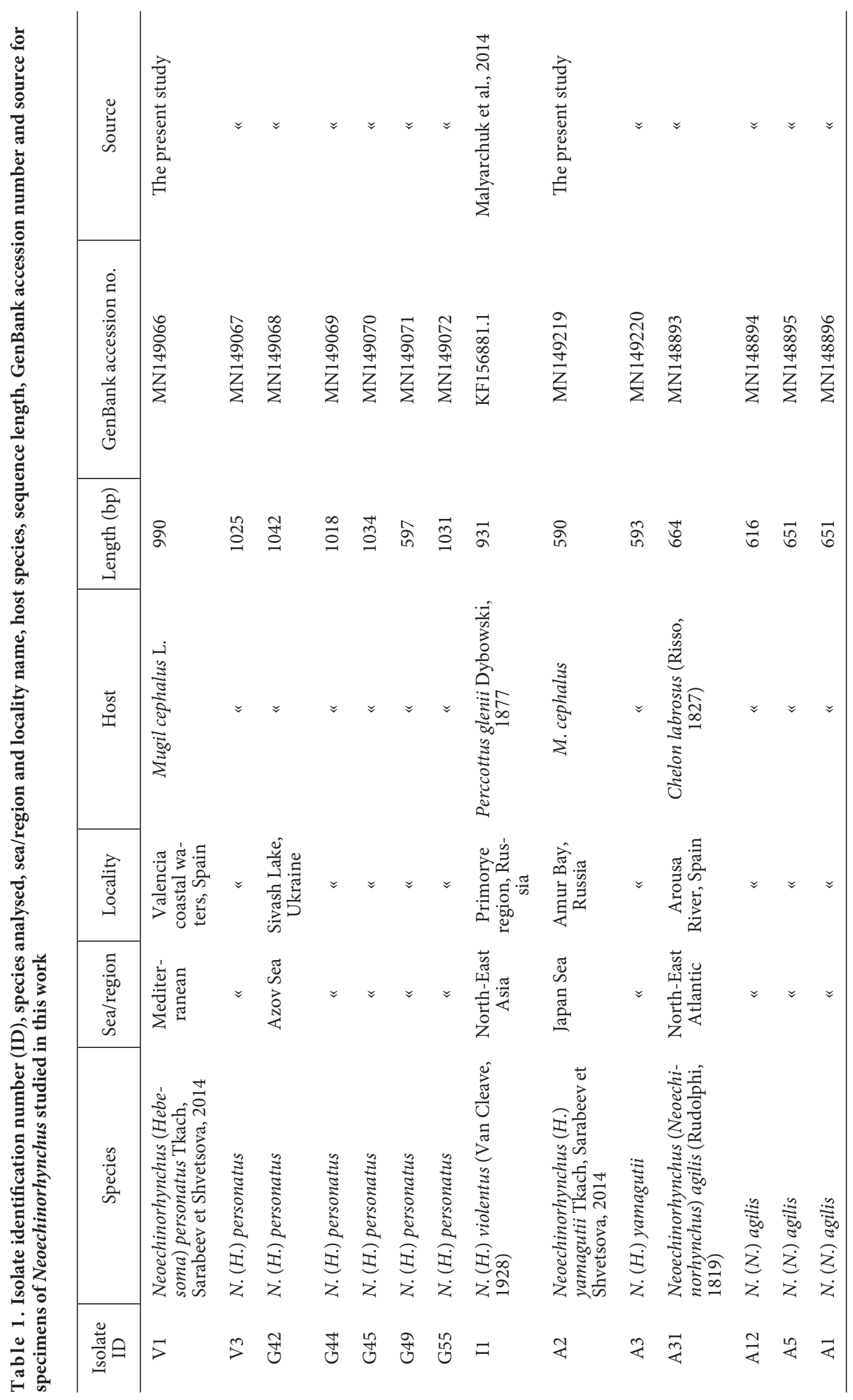


7
0
5
0
\pm
0
0
5
$\Xi$
$\Xi$
5
0
0

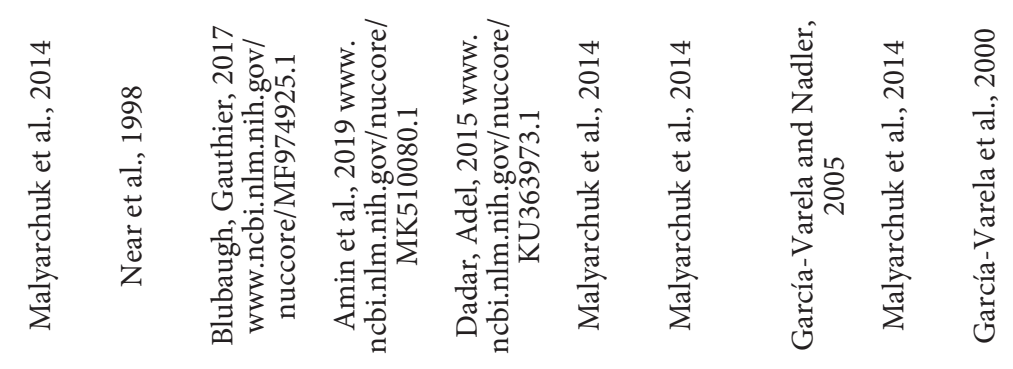

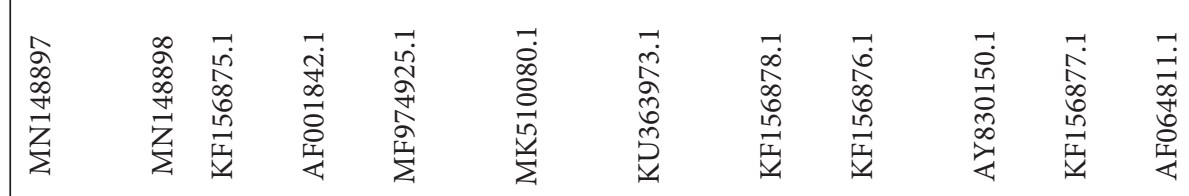

苍

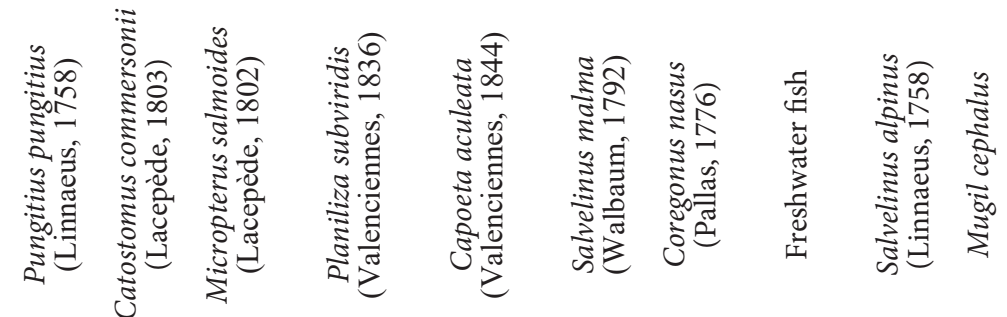

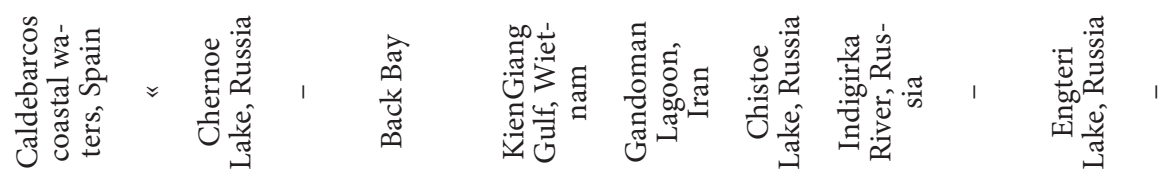

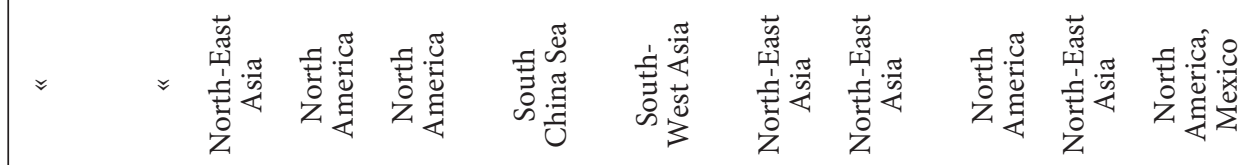

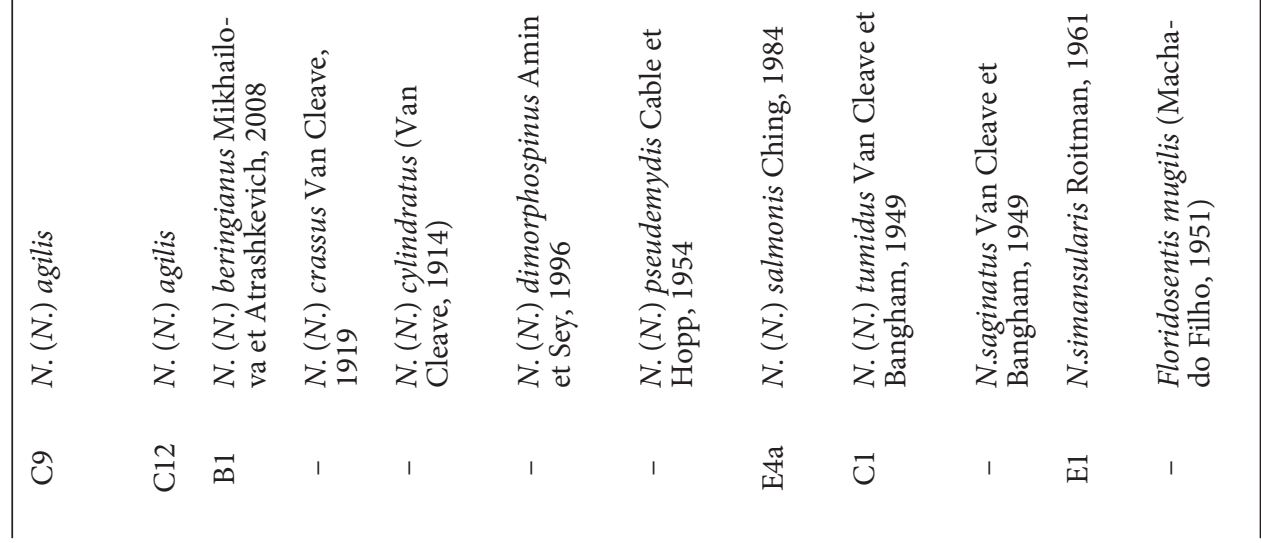


use of the Primer 3Plus program (www.bioinformatics.nl/cgi-bin/primer3plus/primer3plus.cgi), with default parameters.

The PCR mixtures $(25 \mu \mathrm{L})$ contained reaction buffer $\left(10 \mathrm{mMTris}-\mathrm{HCl}, 50 \mathrm{mMKCl}, 1.5 \mathrm{mM} \mathrm{MgCl}_{2}\right)$, $0.2 \mathrm{mM}$ of each deoxynucleoside triphosphate (dNTPs, Roche), $0.4 \mu \mathrm{M}$ of each primer; 0.5 units of high fidelity Taq polymerase and $50 \mathrm{ng}$ of genomic DNA. The reactions were run in an automatic thermocycler (Mastercycler, Eppendorf, Germany) as follows: initial denaturing at $94{ }^{\circ} \mathrm{C}$ for $5 \mathrm{~min}$, followed by 35 cycles at $94{ }^{\circ} \mathrm{C}$ for $30 \mathrm{~s}$, annealing at 57 and $55^{\circ} \mathrm{C}$ (for F-566 and R-1200 and FNeo1086 and RNeo1086 primers, respectively) for $45 \mathrm{~s}$, and $72{ }^{\circ} \mathrm{C}$ for $1 \mathrm{~min}$; and finally, a 7 min extension phase at $72{ }^{\circ} \mathrm{C}$. The PCR products were separated on a $4 \%$ agarose gel in Tris acetate ethylenediaminetetraacetic acid (TAE) buffer containing Sybr Green at $1 \times$ concentration, to verify the presence of bands of the correct size under a variable-intensity UV transilluminator and auto image capture software (Alpha Innotech, USA). The PCR product was purified with Thermo Scientific GeneJETPCR Purification Kit and sequenced in complementary directions using Sanger sequencing service (GATC Biotech, Germany).

The sequences were aligned using Clustal Omega online service (Madeira et al., 2019). All positions containing missing data were eliminated and phylogenetic analyses were performed. Trees were obtained using maximum likelihood (ML) with Tamura-Nei model, neighbour joining (NJ) and minimum evolution (ME) methods as applied in MEGA-X (Kumar et al., 2018). Clade support was assessed by bootstrap resampling with 100 replicates. The tree was drawn to scale, with branch lengths in the same units as those of the evolutionary distances used to infer the phylogenetic tree. The evolutionary distances were computed using the Kimura 2-parameter method (Kimura, 1980).

Estimates of evolutionary divergence based on the number of base differences per site from averaging over all sequence pairs between and within groups (uncorrected p distances) were conducted in MEGA-X and expressed in percentages (table 2). For comparative analysis, the following DNA sequences of Neoechinorhynchus spp. were retrieved from GenBank: N. (N.) beringianus Mikhailova et Atrashkevich, 2008; N. (N.) tumidus Van Cleave and Bangham, 1949; N. simansularis Roitman, 1961; N. (N.) salmonis Ching, 1984; N. (H.) violentus (Van Cleave, 1928); N. saginatus; N. (N.) crassus; N. (N.) pseudemydis; N. (N.) cylindratus; N. (N.) dimorphospinus. The complete $18 \mathrm{~S}$ sequence of Floridosentis mugilis (Machado-Filho, 1951) was used as outgroup based on its sister relationship to Neoechinorhynchus (Malyarchuk et al., 2014; Rosas-Valdez et al., 2012).

\section{Results}

Genetic divergence of Neoechinorhynchus from grey mullets

Seven specimens of $N$. (H.) personatus, 6 of N. (N.) agilis, and 2 of N. (H.) yamagutii were sequenced and used for phylogenetic analysis here (table 1). All species were amplified and sequenced using both universal and specific set of primers (F-566 and R-1200; and FNeo1086 and RNeo1086, respectively), excepting for $N$. (H.) yamagutii for which sequences were obtained only with universal primers. PCR products varied from 590 to $664 \mathrm{bp}$ for

Table 2. Estimates of evolutionary divergence between sequences encoding $18 \mathrm{~S}$ rRA gene of specimens of Neoechinorhynchus from grey mullets across localities in the North-East Atlantic, Mediterranean and Japan Sea

\begin{tabular}{|c|c|c|c|c|c|c|c|c|c|c|}
\hline No & * & 1 & 2 & 3 & 4 & 5 & 6 & 7 & 8 & 9 \\
\hline 1 & N. (H.) yamagutii A2 and A3 & & & & & & & & & \\
\hline 2 & N. (H.) personatus V1 & 6.56 & & & & & & & & \\
\hline 3 & N. (H.) personatus V3 & 6.35 & 0.19 & & & & & & & \\
\hline 4 & N. (H.) personatus G42 & 6.36 & 0.56 & 0.37 & & & & & & \\
\hline 5 & N. (H.) personatus G44 & 6.56 & 0.75 & 0.56 & 0.19 & & & & & \\
\hline 6 & N. (H.) personatus G45, G49 and G55 & 6.16 & 0.37 & 0.19 & 0.19 & 0.37 & & & & \\
\hline 7 & N. (N.) agilis $\mathrm{A} 31$ & 6.57 & 2.85 & 2.66 & 2.66 & 2.85 & 2.47 & & & \\
\hline 8 & N. (N.) agilis A1, A5 and A12 & 6.35 & 2.65 & 2.46 & 2.46 & 2.65 & 2.27 & 0.19 & & \\
\hline 9 & N. (N.) agilis C9 and C12 & 6.15 & 2.46 & 2.27 & 2.27 & 2.46 & 2.08 & 0.37 & 0.19 & \\
\hline
\end{tabular}

Note. Analyses were conducted using the Kimura 2-parameter model (Kimura, 1980) also, the evolutionary rates when the divergence times are known. Uncorrected p distances are shown as percentages. There were a total of $541 \mathrm{bp}$ in the final dataset

* Sequences of A2 and A3 of N. (H.) yamaguti, G45, G49 and G55 of N. (N.) personatus, A1, A5 and A12, and $\mathrm{C} 9$ and $\mathrm{C} 12$ of $N$. (N.) agilis were combined because their $18 \mathrm{~S}$ partial sequences were identical. 
the universal set of primers and from 990 to $1042 \mathrm{bp}$ for specific ones. The aligned data set included 15 sequences with a length of $541 \mathrm{bp}$ and occupying position from 551 to $1092 \mathrm{nu}$ cleotides along the $18 \mathrm{~S}$ rRNA gene of $F$. mugilis. The genetic divergence estimated among specimens of the same species ranged from 0 to $0.75 \%$ (table 2), while distances between taxa of Neoechinorhynchus were much higher (2.08-6.57 \%). The $18 \mathrm{~S}$ sequence divergence between $N$. (H.) yamagutii and both Atlantic species, $N$. (H.) personatus and $N$. (N.) agilis, were higher than that between the latter two (6.15-6.57\% vs. $2.08-2.85 \%$, respectively). A comparison of long sequences of $N$. (H.) personatus and $N$. (N.) agilis (positions from 96 to 550 nucleotides along the $18 \mathrm{~S}$ rRNA gene of $F$. mugilis) supplied additional divergence in $3 \mathrm{bp}$ between these species and revealed difference in 2 bp between specimens of $\mathrm{C} 9$ and C12 of $N$. (N.) agilis, and in 1 bp between G49 and other specimens of N. (H.) personatus.

Phylogenetic analysis of Neoechinorhynchus from grey mullets The phylogenetic analysis of specimens of Neoechinorhynchus from grey mullets revealed that 497 out of 541 characters were constant, 41 were parsimony-informative, and 3 variable characters were parsimony-uninformative. The ML analysis yielded a single tree with the highest log likelihood $=-1242.96$. Trees obtained with NJ and ME methods showed the same topology. The bootstrap support was high for all branches of the phylogenetic tree (fig. 1). All trees comprise of 3 major monophyletic clades, which correspond to $N$. (H.) yamagutii, $N$. (N.) agilis and $N$. (H.) personatus. Clades $N$. (N.) agilis and $N$. (H.) personatus are subsequently subdivided into 2 and 3 subclades, respectively, mainly reflecting different localities.

Phylogenetic relationships of Neoechinorhynchus from grey mullets with other members of the genus

The longest sequence of each species obtained in the present study was combined with published sequences from GenBank, including 10 Neoechinorhynchus species and F. mugilis as outgroup taxa. The aligned data set included 14 sequences, with 442 characters, of which 86 were parsimony-informative. The ML tree with the highest log likelihood (-1619.55) is shown in figure 2 . Trees obtained with NJ and ME methods showed a similar topology. This phylogenetic analysis revealed two main clades with strong bootstrap support. One includes Neoechinorhynchus spp. associated with freshwater and brackish water fishes; another is

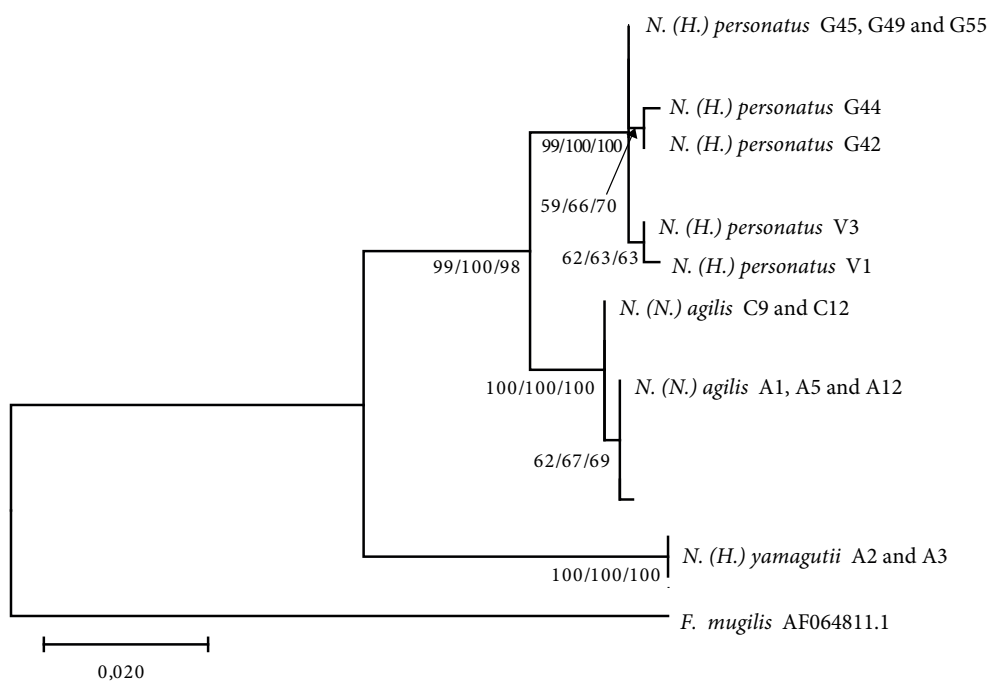

Fig. 1 Phylogenetic tree of Neoechinorhynchus from grey mullets and across localities in the North-East Atlantic, Mediterranean and Japan Sea obtained with maximum likelihood (ML) method ( $\ln$ likelihood 1242.96) based on the $18 \mathrm{~S}$ rRNA partial gene sequences. Floridosentis mugilis was used as an outgroup. The ML/neighborn joining/ minimum evolution bootstrap support is shown at each internal node. Identification number of isolates is as in table 1 . 
composed by $N$. (H.) violentus and monophyletic subclade containing species from marine grey mullets. This subclade formed by the species $N$. (N.) dimorphospinus $-N$. (N.) agilis is phylogenetically distant from other species of the genus and strongly supported by bootstrap values ( $100 \%$ for all methods of tree reconstruction). The bootstrap analysis also supports the sister relationship of $N$. (N.) dimorphospinus and $N$. (H.) yamagutii with the two terminal Atlantic species N. (N.) agilis and N. (H.) personatus. N. (N.) dimorphospinus and $N$. (H.) yamagutii are grouped together in one clade with moderate bootstrap support (49\%) in tree reconstructed by ME method (the result is not shown).

\section{Discussion}

The phylogenetic analysis inferred from partial sequences of the 18S rRNA gene supports the previous morphological observation of Tkach et al. (2014) indicating that $N$. (N.) agilis, $N$. (H.) personatus and $N$. (H.) yamagutii represent three independent species. The genetic divergences estimated between three species sequenced here were relatively high, at the range between $2.08 \%$ and $6.57 \%$. These levels of genetic divergence among species are similar or even higher to those exhibited within Neoechinorhynchus and ranging between $0.37 \%$ and $5.55 \%$ for the $18 \mathrm{~S}$ rRNA gene (Malyarchuk et al., 2014). Our data, consistent with results of Malyarchuk et al. (2014), demonstrate the existence of genetic heterogeneity in the species within Neoechinorhynchus. The closeness of species representing different clades of the phylogenetic tree can be explained by their common origin in a certain geographic region or in evolutionary related definitive hosts. The monophyletic clade formed with species of Neoechinorhynchus from grey mullets, $N$. (N.) dimorphospinus, $N$. (H.) yamagutii, $N$. (N.) agilis and $N$. (H.) personatus, indicates that all these species share a common ancestor. The terminal position of $N$. (N.) agilis and $N .(H$.$) personatus and the$ low genetic divergence found between them suggests the recent emergence of this group and that the colonization of the North-East Atlantic and the Mediterranean regions could represent a single event. One possible explanation of the presence of two closely related species of Neoechinorhynchus in the Mediterranean is an evolutionary host-switching, as $N$. (N.) agilis and N. (H.) personatus tend to parasitize two different host species, C. labrosus and M. cephalus, respectively (Tkach et al., 2014). Geographic barriers, such as land masses of Africa and India, prevent migration and consequent gene flow among acanthocephalans,

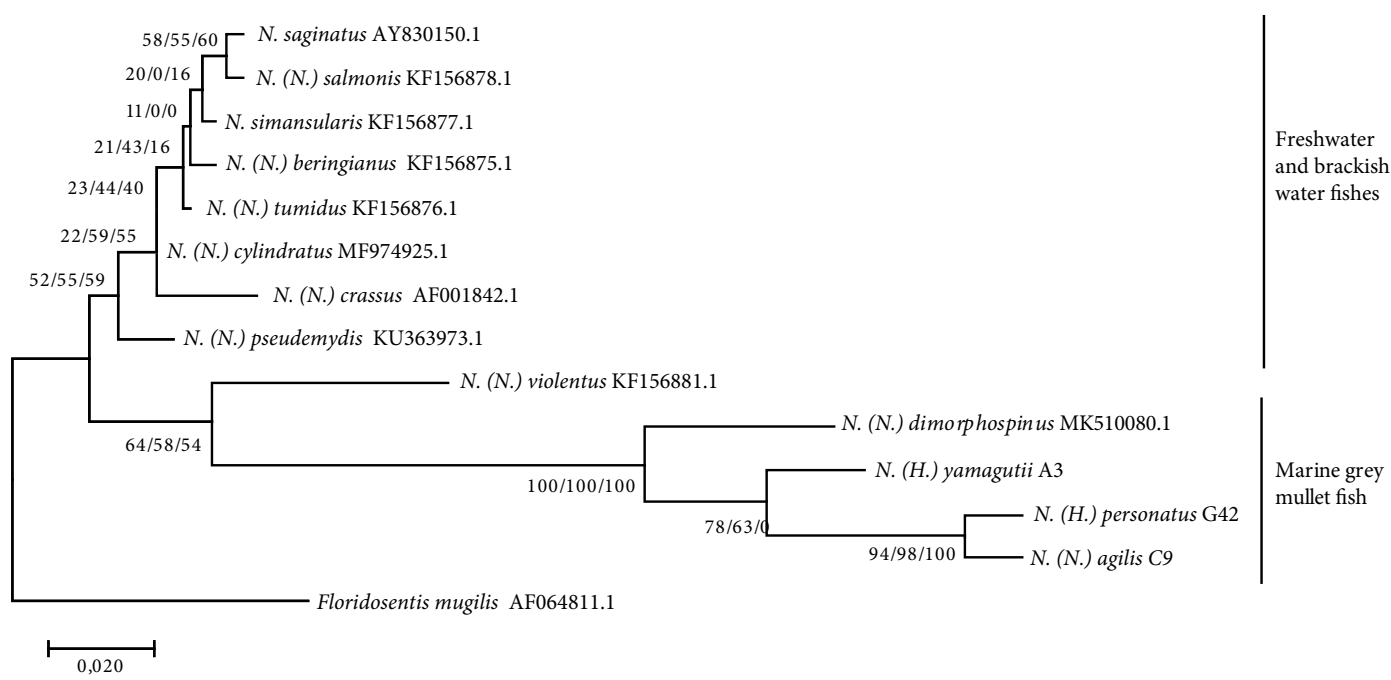

Fig. 2 Phylogenetic tree of Neoechinorhynchus species obtained with maximum likelihood (ML) method (- ln likelihood 1619.55) based on the 18S rRNA partial gene sequences. Floridosentis mugilis was used as an outgroup. The ML/neighborn joining/minimum evolution bootstrap support is shown at each internal node. 
may explain a high divergence level between $N$. (H.) yamagutii and both Atlantic species, $N$. (N.) agilis and $N$. (H.) personatus. The basal position of $N$. (N.) dimorphospinus and $N$. (H.) yamagutii in relation to $N$. (N.) agilis and $N$. $(H$.$) personatus indicates that the$ origin of the ancestor of the latter ones, most likely, related with colonization of the NorthEast Atlantic region by the Pacific species with subsequent vicariance event. Our results suppose that colonization of $N$. (H.) yamagutii of the Sea of Japan is associated with grey mullet fish rather than with freshwater and brackish water fishes of North-East Asia, since the species of Neoechinorhynchus from these groups of hosts represent different lineages.

Although Hadziavdic et al. (2014) suggested that acanthocephalans could not be amplified using the primer pair F-566 and R-1200, the PCR product and sequences for all three species of Neoechinorhynchus studied here were successfully obtained by applying this set of universal primers. The amplicons of acanthocephalan individuals generated using this universal set of primers averaged 623 nucleotides in length, whereas the specific primer pair supplied fragments with the average length in 1021 nucleotides covering the variable regions V4-V5 and V2-V5 of the $18 \mathrm{~S}$ rRNA gene, respectively. In agreement with the results of Hadziavdic et al. (2014), our study demonstrates that the variable region V4$\mathrm{V} 5$ yielded higher taxonomic information than the region V2-V3 providing 11 informative characters vs. 3 when comparing sequences of $N$. (N.) agilis and N. (H.) personatus. Thus, the universal primer pair F-566 and R-1200 can be effectively used to amplify acanthocephalans for assessing their diversity in field-based studies.

Another important finding of the present study is that the subgenus Hebesoma is the polyphyletic group. While all three species of the subgenus Hebesoma are assigned to one clade, two species of the subgenus Neoechinorhynchus are nested within the same clade (see fig. 2). In addition, the genetic divergence was higher between $N$. (H.) yamagutii and $N$. (H.) personatus than between the latter species and $N$. (N.) agilis. These results match those inferred from CO1 gene by Malyarchuk et al. (2014) for the speciesof Neoechinorhynchus and Hebesoma from North-East Asia.Van Cleave (1928) erected the genus and the family Hebesomidae for Hebesoma violentum. In the diagnosis of the species he indicated that the eggs had globular polar enlargements of middle membrane, lemnisci were short, and the giant subcuticular nuclei were unnoticeable. Meyer (1932) transferred Hebesoma to Neoechinorhynchidae, leaving diagnoses of the genus, and of $H$. violentum without changes. The validity of Hebesoma was accepted by Petrochenko (1956) and Yamaguti (1963), they both provided more concise diagnosis of the genus. Salgado-Maldonado (1978) dismissed the importance of the features listed in Hebesoma diagnosis, showing their presence in the descriptions of many Neoechinorhynchus species and proposed the synonymy of these genera. Amin (2002) argued against monophyly of Neoechinorhynchus. He proposed to combine the concepts of both genera and to lower the status of the Neoechinorhynchus (sensu stricto) and Hebesoma emphasizing on subgeneric importance of the egg structure. Neoechinorhynchus is characterized by uniformity of anatomical organization (Malyarchuk et al., 2014), while the specific egg morphology observed in some species provides an important morphologic feature useful for species discrimination. At the same time, erection of a species group to a higher level taxon based on the polar prolongations of the inner envelopes of an egg is not consistent with the molecular results arguing that Hebesoma is supposed to be an unnatural taxon.

In conclusion, morphologically similar species $N$. (N.) agilis and $N$. (H.) personatus and $N$. (H.) yamagutii can be clearly recognized using universal primers (F-566 and R-1200) proposed by Hadziavdic et al. (2014) for 18 S rRNA gene. Phylogenetic analysis demonstrates that the studied here species of Neoechinorhynchus from grey mullet fish share common ancestor, despite their different geographic location, and are closely related. Hebesoma is shown to be a polyphyletic taxon. In future investigations, it might be possible to use additional genomic markers to assess the validity of the phylogenetic hypotheses inferred in the present study. 
We are grateful to Raúl Míguez-Lozano from University of Valencia for providing specimens of N. (H.) personatus from Valencia coastal waters, Spain and Lyudmila Shvetsova from Pacific Research Fisheries Center for providing specimens of $N$. (H.) yamagutii from Amur Bay, Russia. This study is supported by MEDEA project of Erasmus Mundus Action 2, \#2686 and partially by an Assemble + short-term fellowship \#BA200119, Ministry of Education and Science of Ukraine (projects \#1/17 and 1/19).

\section{Author contributions}

IeT, JL and VS conceived the ideas. IeT collected the data from the Azov Sea, VS from the North-East Atlantic. RAS designed methodology of DNA extraction and amplification. JL designed primers set. VS obtained PCR product. JL and VS analysed the data. VS led the writing and IeT contributed to the writing of the manuscript. All authors contributed critically to the drafts and gave final approval for publication.

\section{References}

Amin, O. M. 2002. Revision of Neoechinorhynchus Stiles \& Hassall, 1905 (Acanthocephala: Neoechinorhynchidae) with keys to 88 species in two subgenera. Syst. Parasitol., 53, 1-18.

Amin, O. M. 2013. Classification of the acanthocephalan. Folia Parasitol. (Praha), 60, 273-305.

De Felipe, A.-P., Lamas, J., Sueiro, R.-A., Folgueira, I., Leiro, J.-M. 2017. New data on flatfish scuticociliatosis reveal that Miamiensis avidus and Philasterides dicentrarchi are different species. Parasitology, 144, 13941411. https://doi.org/10.1017/S0031182017000749

García-Varela, M., Nadler, S. A. 2005. Phylogenetic relationships of Palaeacanthocephala (Acanthocephala) inferred from SSU and LSU rDNA gene sequences. J. Parasitol., 91, 1401-1409. https://doi.org/10.1645/ GE-523R.1

García-Varela, M., Pérez-Ponce de León, G., de la Torre, P., Cummings, M. P., Sarma, S. S. S., Laclette, J. P. 2000. Phylogenetic relationships of Acanthocephala based on analysis of $18 \mathrm{~S}$ ribosomal RNA gene sequences. J. Mol. Evol., 50, 532-540. https://doi.org/10.1007/s002390010056

Gautam, N. K., Mohapatra, A., Saxena, A. M. 2018. On a new species of Neoechinorhynchus Hamann, 1892 (Neoechinorhynchinae Travassos, 1926) from Eleutheronema tetradactylum (Shaw, 1804) from Digha coast, West Bengal, India. J. Parasit. Dis., 42, 462-466. https://doi.org/10.1007/s12639-018-1019-6

Hadziavdic, K., Lekang, K., Lanzen, A., Jonassen, I., Thompson, E. M., Troedsson, C. 2014. Characterization of the 18S rRNA Gene for Designing Universal Eukaryote Specific Primers. PLoS One, 9, e87624. https://doi. org/10.1371/journal.pone.0087624

Hebert, P. D. N., Cywinska, A., Ball, S. L., DeWaard, J. R. 2003. Biological identifications through DNA barcodes. Proc. R. Soc. London. Ser. B Biol. Sci., 270, 313-321. https://doi.org/10.1098/rspb.2002.2218

Kimura, M. 1980. A simple method for estimating evolutionary rates of base substitutions through comparative studies of nucleotide sequences. J. Mol. Evol., 16, 111-120.

Kumar, S., Stecher, G., Li, M., Knyaz, C., Tamura, K. 2018. MEGA X: Molecular Evolutionary Genetics Analysis across Computing Platforms. Mol. Biol. Evol., 35, 1547-1549. https://doi.org/10.1093/molbev/msy096

Leiro, J., Siso, M.I., Parama, A., Ubeira, F. M., Sanmartin, M. L. 2000. RFLP analysis of PCR-amplified small subunit ribosomal DNA of three fish microsporidian species. Parasitology, 120, 113-119.

Madeira, F., Park, Y. M., Lee, J., Buso, N., Gur, T., Madhusoodanan, N., Basutkar, P., Tivey, A. R. N., Potter, S. C., Finn, R. D., Lopez, R. 2019. The EMBL-EBI search and sequence analysis tools APIs in 2019. Nucleic Acids Res. https://doi.org/10.1093/nar/gkz268

Malyarchuk, B., Derenko, M., Mikhailova, E., Denisova, G. 2014. Phylogenetic relationships among Neoechinorhynchus species (Acanthocephala: Neoechinorhynchidae) from North-East Asia based on molecular data. Parasitol. Int., 63, 100-107. https://doi.org/10.1016/j.parint.2013.09.012

Melo, F. T. V., Costa, P. A. F. B., Giese, E. G., Gardner, S. L., Santos, J. N. 2015. A description of Neoechinorhynchus (Neoechinorhynchus) veropesoi $\mathrm{n}$. sp. (Acanthocephala: Neoechinorhynchidae) from the intestine of the silver croaker fish Plagioscion squamosissimus (Heckel, 1840) (Osteichthyes: Sciaenidae) off the east coa. J. Helminthol., 89 (1), 34-41. https://doi.org/10.1017/S0022149X13000564

Meyer, A. 1932. Acanthocephala. In: Bronns, H. G., ed. Klassen Und Ordnungen Des Tierreichs, Vierter Band, 2nd Abteilung. Akademische Verlagsgesellschaft M. B. H., Leipzig, 1-332.

Morand, S. 2018. Advances and challenges in barcoding of microbes, parasites, and their vectors and reservoirs. Parasitology, 145, 537-542. https://doi.org/10.1017/S0031182018000884

Near, T. J., Garey, J. R., Nadler, S. A. 1998. Phylogenetic relationships of the acanthocephala inferred from 18S ribosomal DNA sequences. Mol. Phylogenet. Evol., 10, 287-298. https://doi.org/10.1006/mpev.1998.0569

Petrochenko, V. I. 1956. Acanthocephala of domestic and wild animals, Vol. 1. Izdatelstvo Akademii Nauk S.S.S.R., Moskow, 1-435.

Pinacho-Pinacho, C. D., Sereno-Uribe, A. L., García-Varela, M. 2014. Morphological and molecular data reveal a new species of Neoechinorhynchus (Acanthocephala: Neoechinorhynchidae) from Dormitator maculatus in the Gulf of Mexico. Parasitol. Int., 63, 763-771. https://doi.org/10.1016/j.parint.2014.07.003 
Rosas-Valdez, R., Morrone, J. J., García-Varela, M. 2012. Molecular phylogenetics of Floridosentis Ward, 1953 (Acanthocephala: Neoechinorhynchidae) parasites of mullets (Osteichthyes) from Mexico, Using $28 \mathrm{~S}$ rDNA Sequences. J. Parasitol., 98, 855-862. https://doi.org/10.1645/GE-2963.1

Salgado-Maldonado, G. 1978. Acantocéfalos de peces IV. Descripción de dos especiesnuevas de Neoechinorhynchus Hamann, 1892 (Acanthocephala: Neoechinorhynchidae) y algunasconsideracioness obreestegénero. An. Inst. Biol. Univ. Nac. Auton. Mex. Ser. Zool., 1, 35-48.

Smales, L. R. 2013. A Review of the Genus Neoechinorhynchus (Acanthocephala: Neoechinorhynchidae) from Australia with the Description of Two New Species. J. Parasitol., 99, 1106-1112. https://doi. org/10.1645/13-184.1

Tkach, I., Sarabeev, V., Shvetsova, L. 2014. Taxonomic status of Neoechinorhynchus agilis (Acanthocephala, Neoechinorhynchidae), with a description of two new species of the genus from the Atlantic and Pacific mullets (Teleostei, Mugilidae). Vestnik Zoologii, 48 (4), 291-306. https://doi.org/10.2478/vzoo-2014-0035

Van Cleave, H. J. 1928. Acanthocephala from China. I. New Species and New Genera from Chinese Fishes. Parasitology, 20, 1-9. https://doi.org/10.1017/S0031182000011409

Yamaguti, S. 1963. Systema helminthum. Vol. V, Acanthocephala. Interscience, London, 1-423.

Received 15 July 2019

Accepted 25 October 2019 